ص ص ص

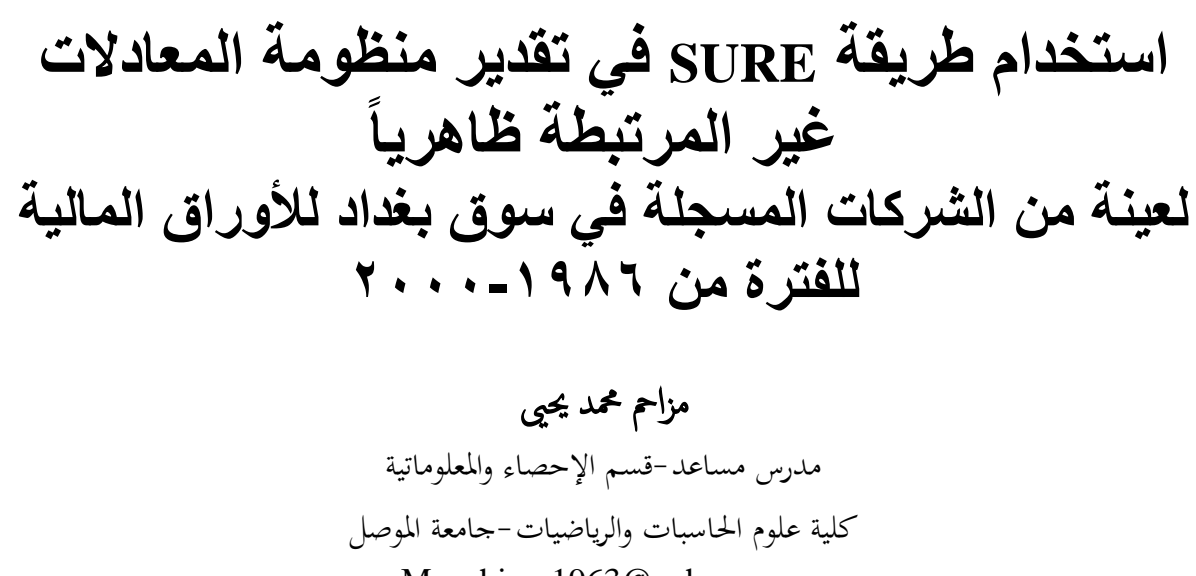

Muzahim_1963@yahoo.com

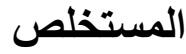

تم في هذا البحث توصيف معادلات ست شركات مختارة من الشركات المسجلة في سوق مقاد

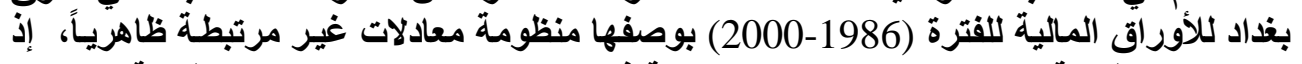

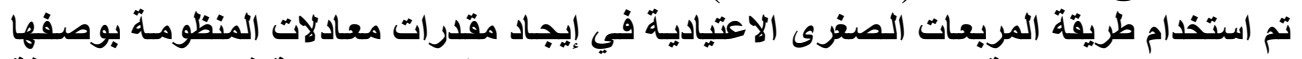

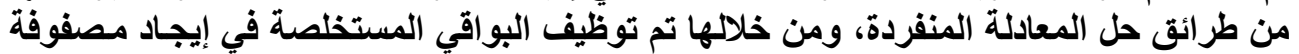

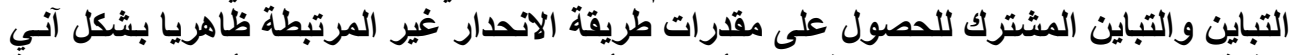

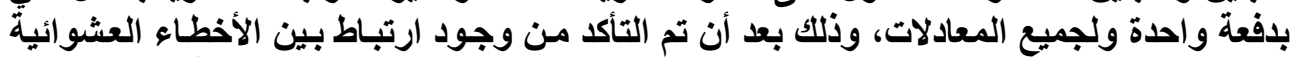

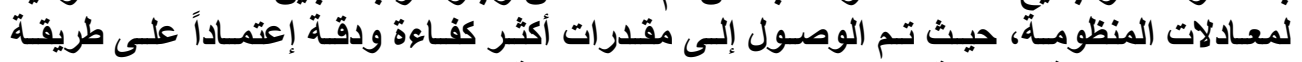

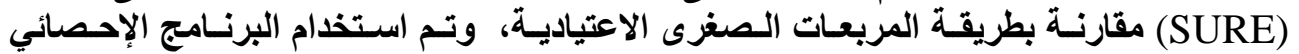
الجاهز (SAS.9) في الحصول بطل على النتائج.




\title{
Using SURE Method for Estimating Seemingly Unrelated Regression Equations Systems for Sampling Registered Companies in the Baghdad Stock Exchange's for the period (1986-2000)
}

\author{
Mozahim M. Yehya \\ Assistant Lecturer \\ Department of Statistics and Information \\ University of Mosul
}

\begin{abstract}
Equations of six selected registered companies have been specified in Baghdad Stock Exchange's for the period during (1986 - 2000), that is Seemingly Unrelated Regression Equations (SURE) system. Ordinary Least Square (OLS) method has been used to estimate the parameter of the equations of the system in a separate way. It is regarded that it is from methods of solving single equation, and from the residuals for this method, the variance - covariance matrix have been obtained by the estimator of Seemingly Unrelated Regression to evaluate the parameters simultaneously in one batch for all the equations, and we reached an efficient and accuracy estimators for the equations of the system comparing with (OLS) method by employing the statistics package (SAS.9).
\end{abstract}

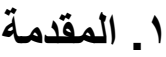

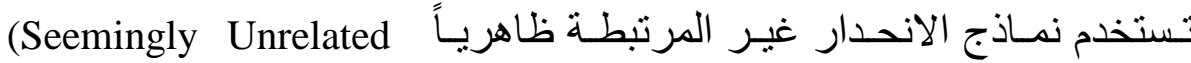

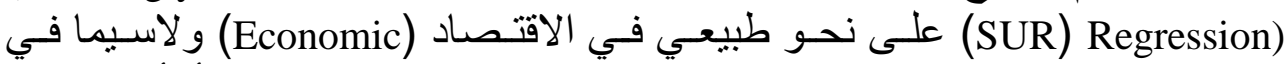

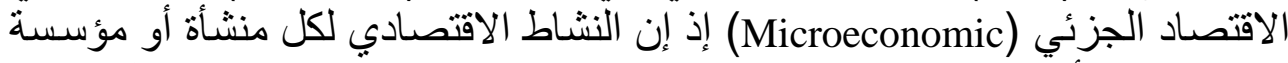

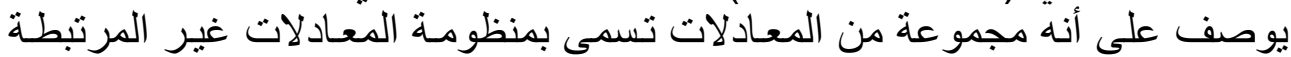

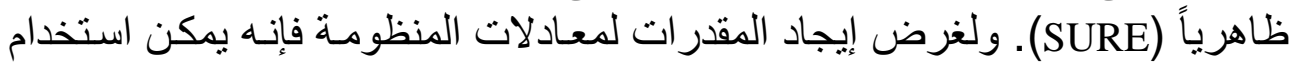

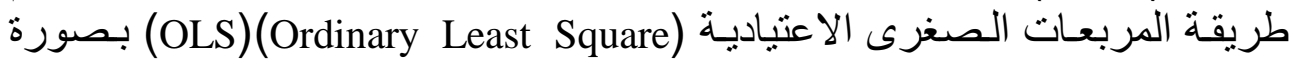

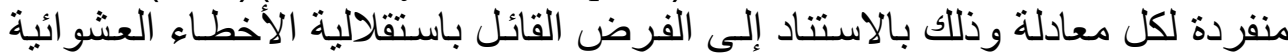

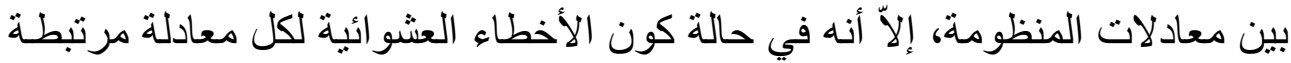

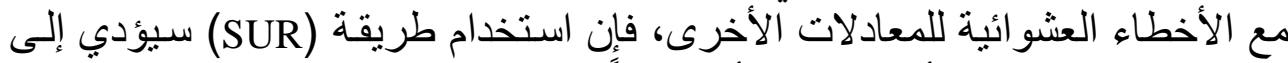

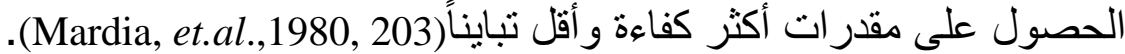

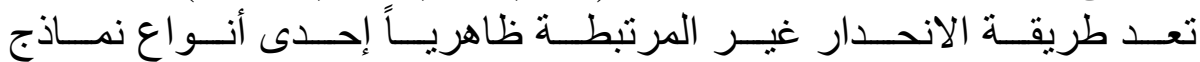

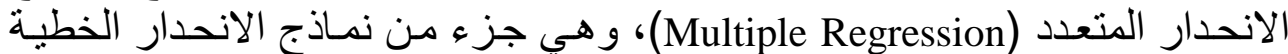
النها (Linear Regression)

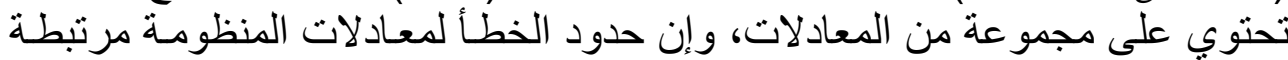

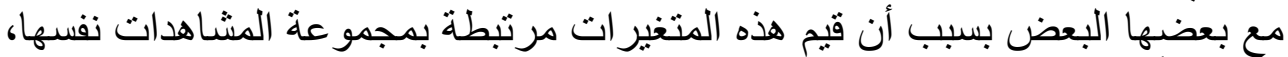

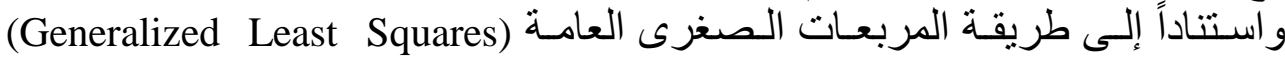

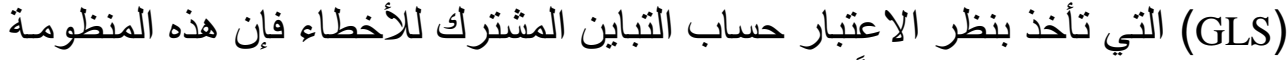

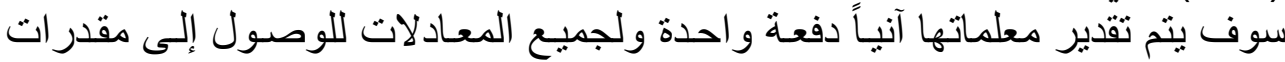




\section{يحيى [rrV]}

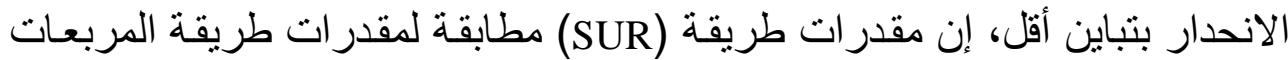

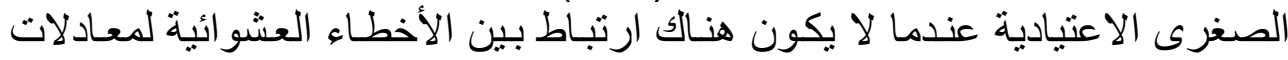

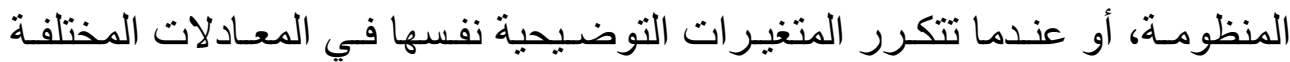

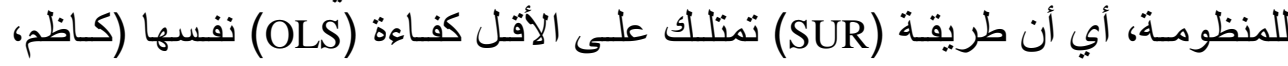

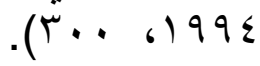

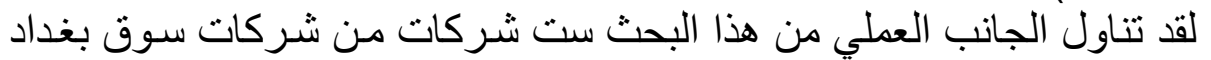

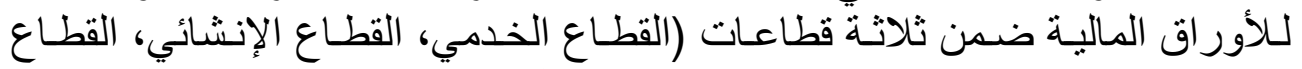

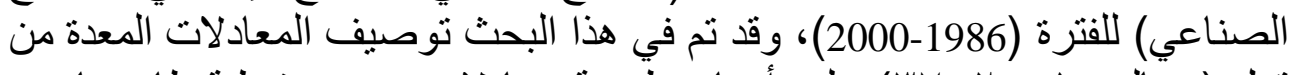

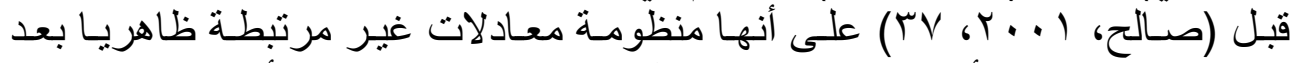

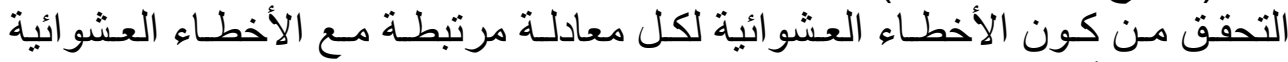

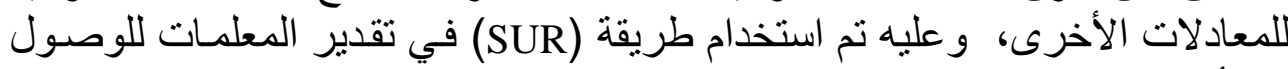
إلى أفضل تعبير قياسي للعلاقة الاقتصادية قيد البحث.

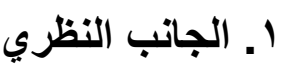

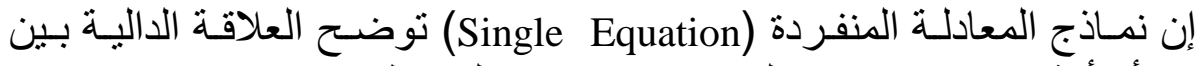

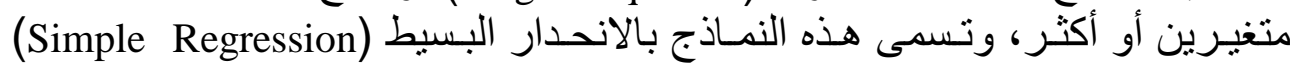

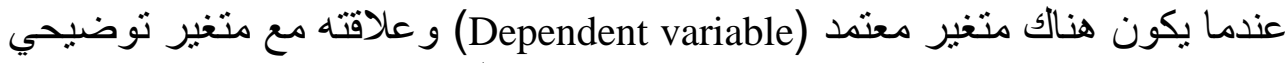
(Multiple Regression) واحد، وتسمى بالانحدار المتعدير (Explanatory Variable)

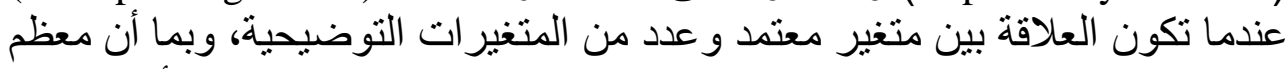

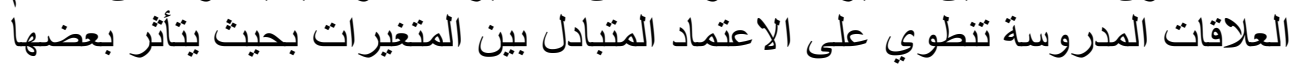

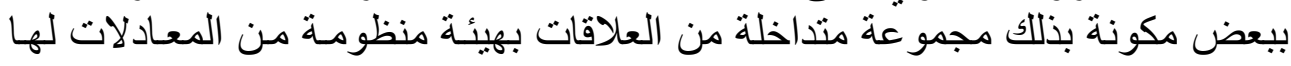

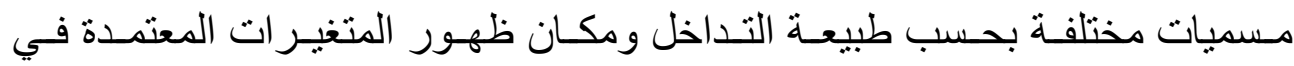

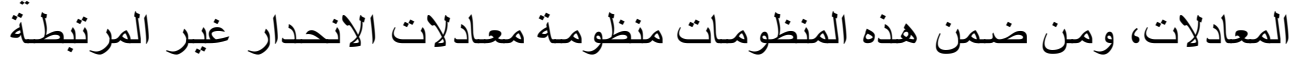

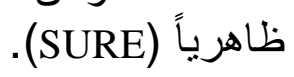

\section{1 - 1 منظومة معادلات الاتحدار غير المرتبطة ظاهريا}

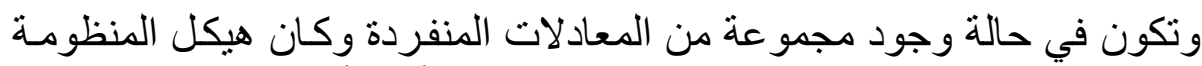

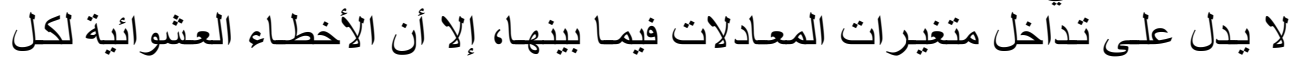

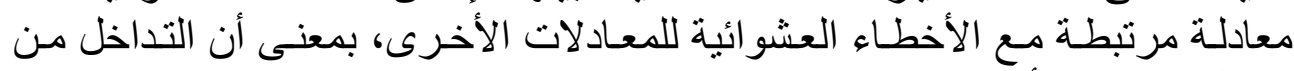

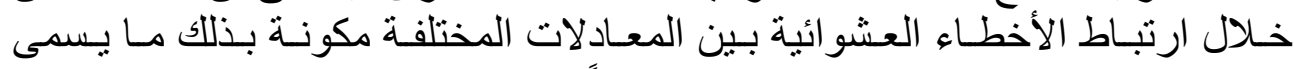

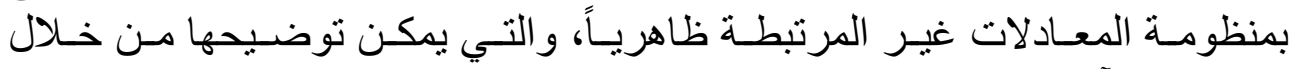
المنظومة الآتية (Pindyack \& Rubinfeld, 1981, 323):

$$
\begin{aligned}
& Y_{1}=a_{0}+a_{1} X_{1}+a_{2} X_{2}+e_{1} \\
& Y_{2}=b_{0}+b_{1} X_{3}+b_{2} X_{4}+e_{2}
\end{aligned}
$$


$Y_{3}=c_{0}+c_{1} X_{5}+c_{2} X_{6}+e_{3}$

يلاحظ من المنظومة المذكورة آنفاً أن أي متغير توضيحي لا يظهر في أكثر

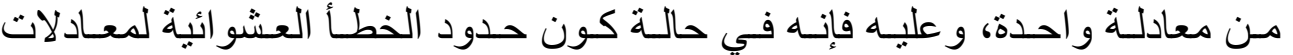

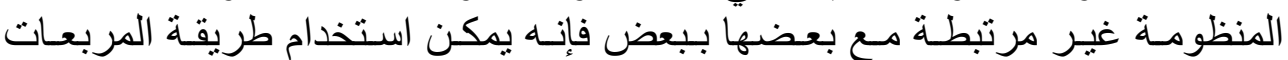

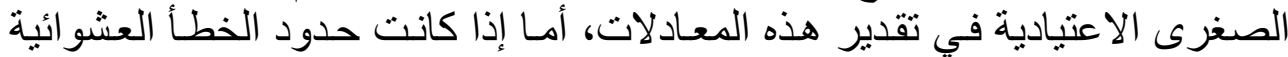

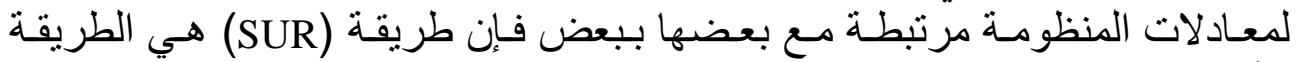
الأكثر كفاءة في التقدير.

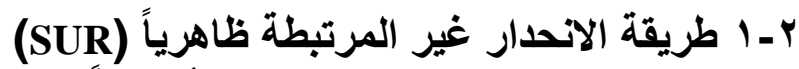

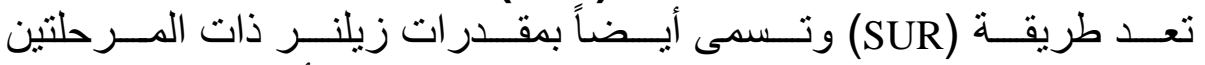
(Mardia, et.al.,1980, 204) (Zellner's two-stage estimator)

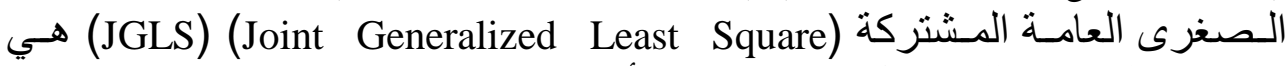

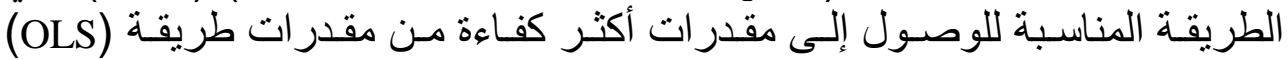

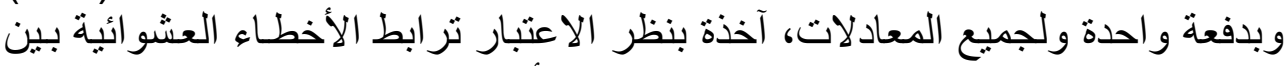

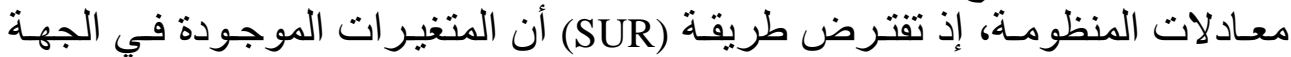

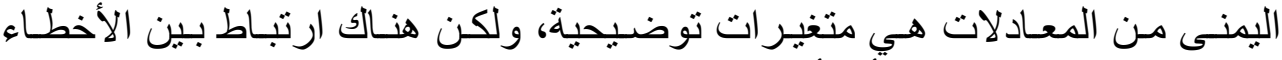
العشو ائية لهذه المعادلات، أي أن:

$\operatorname{cov}\left(e_{1}, e_{2}\right) \neq \operatorname{cov}\left(e_{1}, e_{3}\right) \neq \operatorname{cov}\left(e_{2}, e_{3}\right) \neq 0$

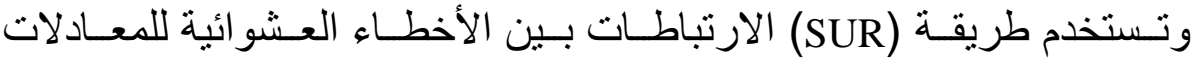

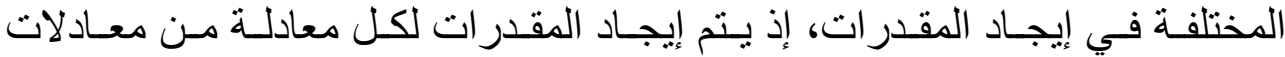

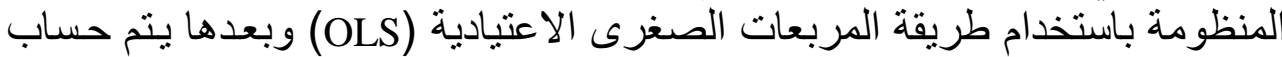

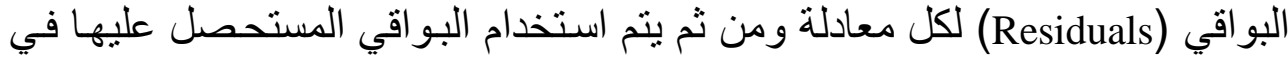

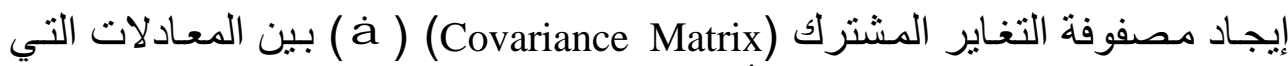

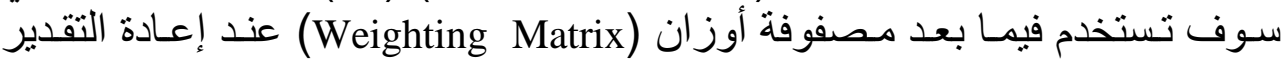

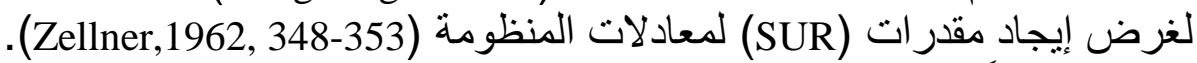

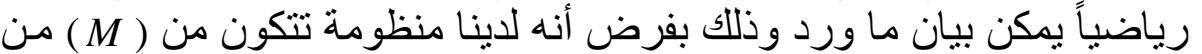
المعادلات، إذ يمكن كتابة المعادلة ( ب ) بالصيغة الآتية: $y_{\mu}=X_{\mu} \beta_{\mu}+u_{\mu} \quad \ldots$

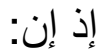

T: (T*k) لمشاهدات المتغير ات التوضيحية في المعادلة.

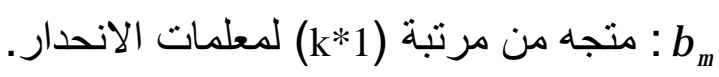

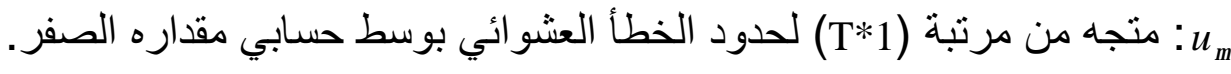
ومن ثم يمكن كتابة المنظومة التي أحد معادلاتها المعادلة 5 بالصيغة الآتية: 


\section{يحيى [Y [Y]}

$\left[\begin{array}{l}y_{1} \\ y_{2} \\ \cdot \\ \cdot \\ \cdot \\ y_{M}\end{array}\right]=\left[\begin{array}{llll}X_{1} & 0 & \ldots & 0 \\ 0 & X_{2} & \ldots & 0 \\ \cdot & \cdot & & \cdot \\ \cdot & \cdot & & \cdot \\ \cdot & \cdot & & \cdot \\ 0 & 0 & \ldots & X_{M}\end{array}\right]\left[\begin{array}{l}\beta_{1} \\ \beta_{2} \\ \cdot \\ \cdot \\ \beta_{M}\end{array}\right]+\left[\begin{array}{l}u_{1} \\ u_{2} \\ \cdot \\ \cdot \\ \cdot \\ u_{M}\end{array}\right] \ldots$

$y=X \beta+u$

X إذ إن

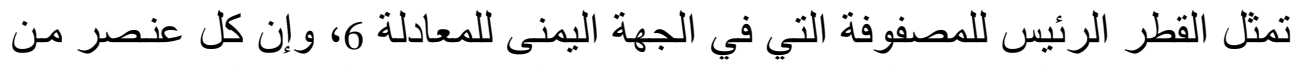

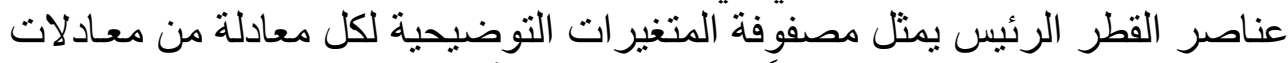

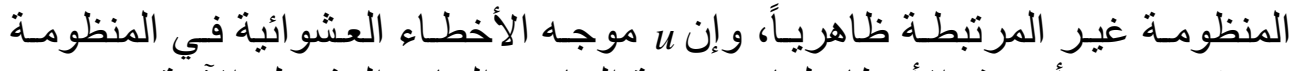

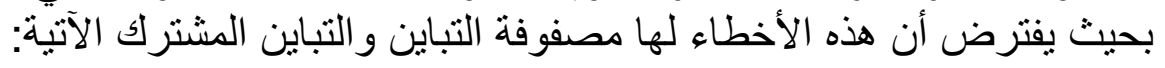

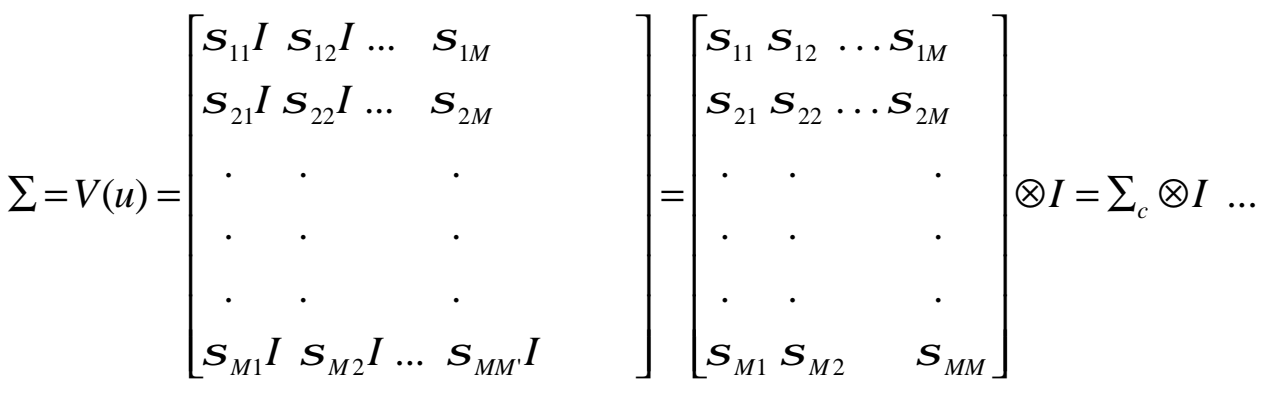

إذ إن Iصفوفة الوحدة من مرتبة I*T.

$$
\sigma_{\mu \mu^{\prime}}=E\left(u_{\mu t} u_{\mu^{\prime} t}\right) \quad t=1,2, \ldots, T
$$

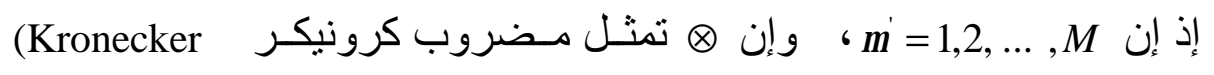

.Product)

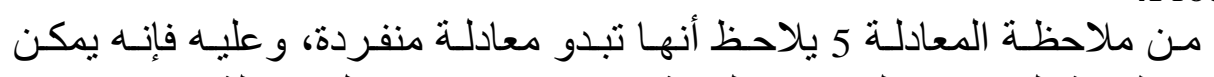
استخدام طريقة المربعات الصغرى العامة في إيجاد مقدر ات كل معادلة من معادلات

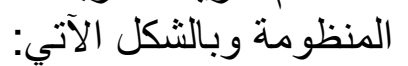

$$
b=\left(X^{\prime} \Sigma^{-1} X\right)^{-1} X^{\prime} \Sigma^{-1} y
$$


$\Sigma^{-1}=V^{-1}(u)=\left[\begin{array}{ccc}\sigma^{11} I & \ldots & \sigma^{1 M} I \\ \cdot & \cdot \\ \cdot & \cdot \\ \cdot & \cdot \\ \sigma^{M 1} I \ldots & \sigma^{M M} I\end{array}\right]=\Sigma_{c}^{-1} \otimes I$

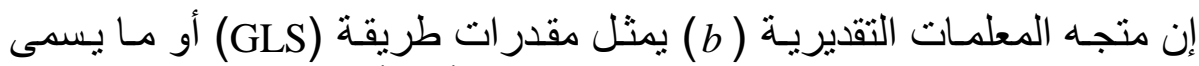

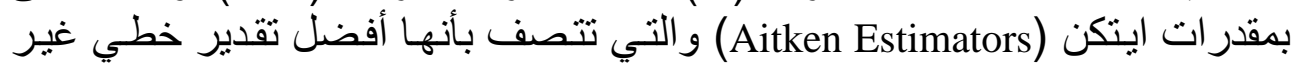
متحيز (BLUE) (Best Linear Unbiased Estimator)

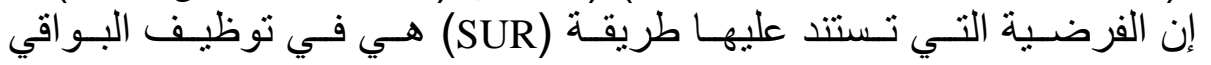

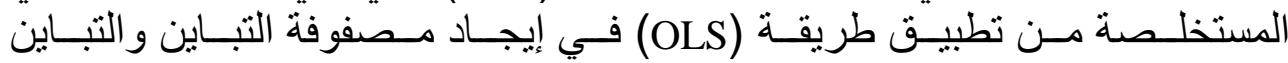

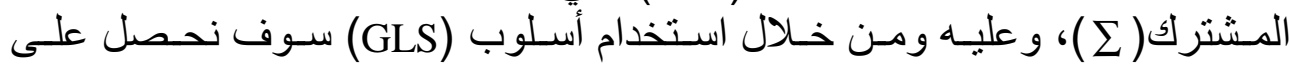

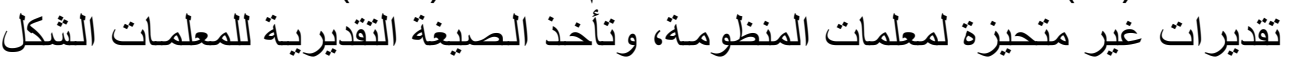
$b=\left[\begin{array}{c}b_{1} \\ b_{2} \\ \cdot \\ \cdot \\ b_{M}\end{array}\right]=\left[\begin{array}{ccc}\sigma^{11} X_{1}^{\prime} X_{1} & \sigma^{12} X_{1}^{\prime} X_{2} \ldots & \sigma^{1 M} X_{1}^{\prime} X_{M} \\ \sigma^{21} X_{2}^{\prime} X_{1} & \sigma^{22} X_{2}^{\prime} X_{2} \ldots & \sigma^{2 M} X_{2}^{\prime} X_{M} \\ \cdot & \cdot & \cdot \\ \cdot & \cdot & \cdot \\ \cdot & \cdot & \cdot \\ \sigma^{M 1} X_{M}^{\prime} X_{1} & \sigma^{M 2} X_{M}^{\prime} X_{2} \ldots & \sigma^{M M} X_{M}^{\prime} X_{M}\end{array}\right]^{-1}\left[\begin{array}{c}\sum_{\mu=1}^{M} \sigma^{1 \mu} X_{1}^{\prime} y_{\mu} \\ \cdot \\ \cdot \\ \cdot \\ \sum_{\mu=1}^{M} \sigma^{M \mu} X_{M}^{\prime} y_{\mu}\end{array}\right] \ldots$

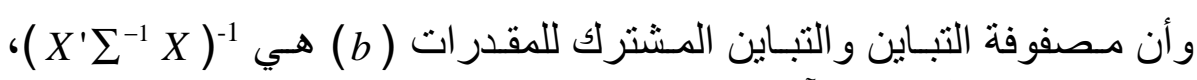
ويمكن التعبير عنها بالصيغة الآتية:

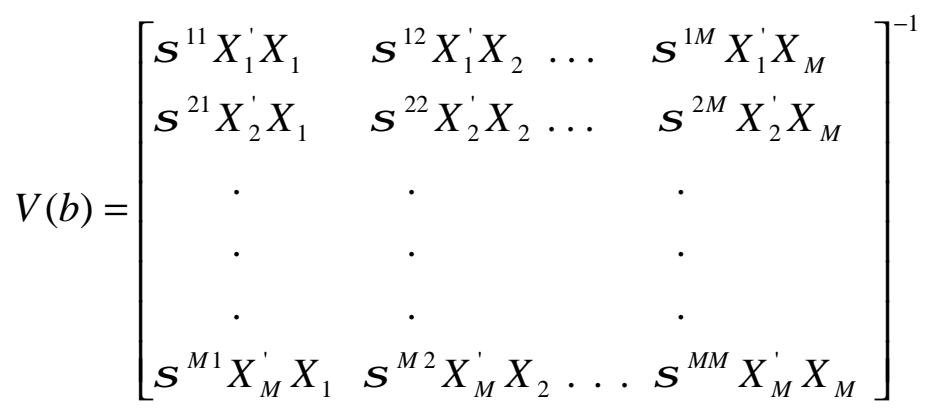

تتصف مقدر ات طريقة (SUR) بأنها أكثر كفاءة من مقدرات طريقة المربعات

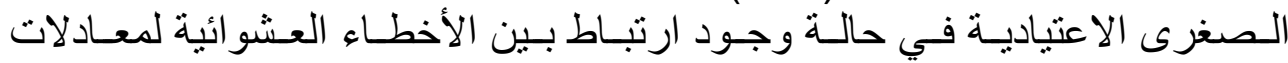




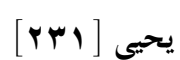

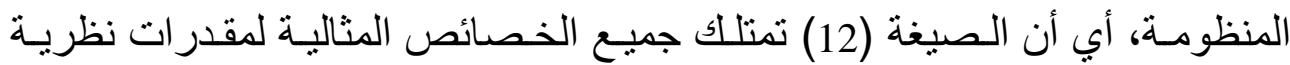

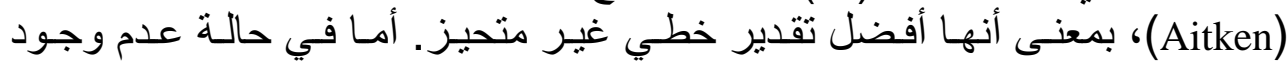

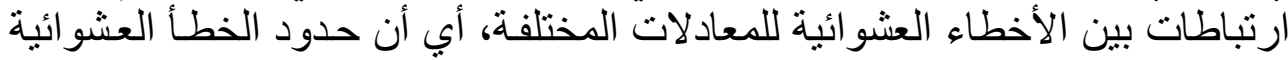

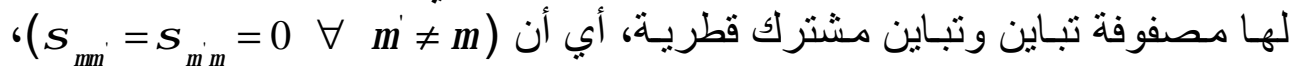

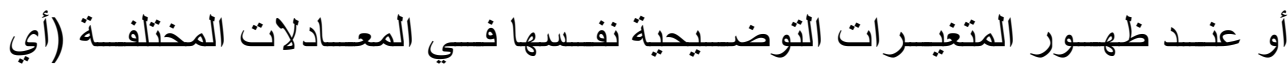
أن أن

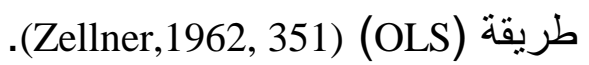

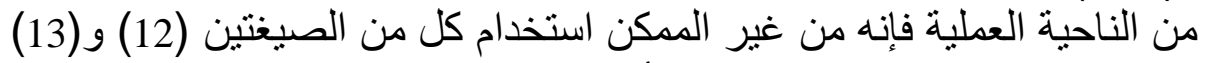

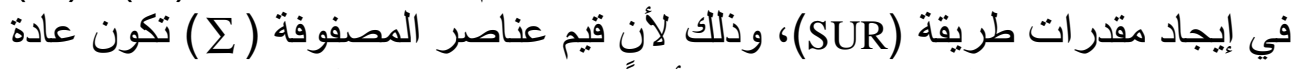

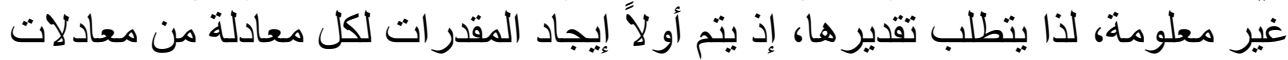
المنظومة باستخدام طريقة (OLS) وكالاتي:

$\hat{B}_{\mu}=\left(X_{\mu}^{\prime} X_{\mu}\right)^{-1} X_{\mu}^{\prime} Y \quad$ -

إذ إن ( OLS) (OL) تمثل مقدر ات طريقة

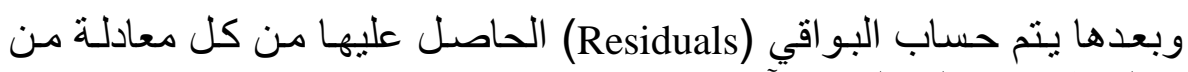

$\hat{u}^{\prime}=y_{\mu}-X_{\mu} \hat{B}_{\mu}$ معادلات المنظومة و على النحو الآتي:

ومن ثم يتم استخدام البو اقي في عملية تقدير عناصر المصفوفة (5) و على النحو الآتي: - ne

$E\left(\hat{u}_{\mu}^{\prime} \hat{u}_{\mu}\right)=E\left\{\left(y_{\mu}-X_{\mu} \hat{B}_{\mu}\right)^{\prime}\left(y_{\mu^{\prime}}-X_{\mu^{\prime}} \hat{B}_{\mu^{\prime}}\right)\right\}$

و التي سوف تستخدم فيما بعد مصفوفة أوزان (Weighting Matrix) عند إعادة التقدير لغرض إيجاد مقدرات طريقة (SUR) وبالثكل الآتي: $\hat{b}=\left(X^{\prime} \hat{\Sigma}^{-1} X\right)^{-1}\left(X^{\prime} \hat{\Sigma}^{-1} Y\right)$

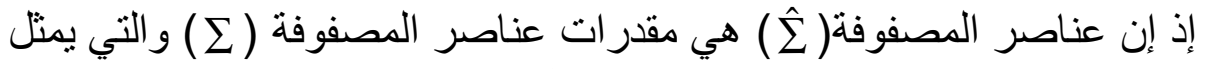

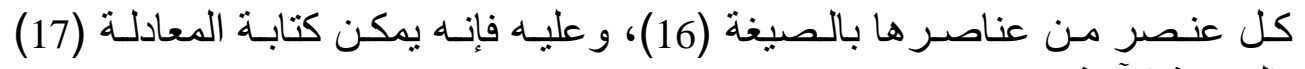

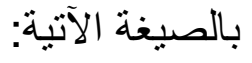




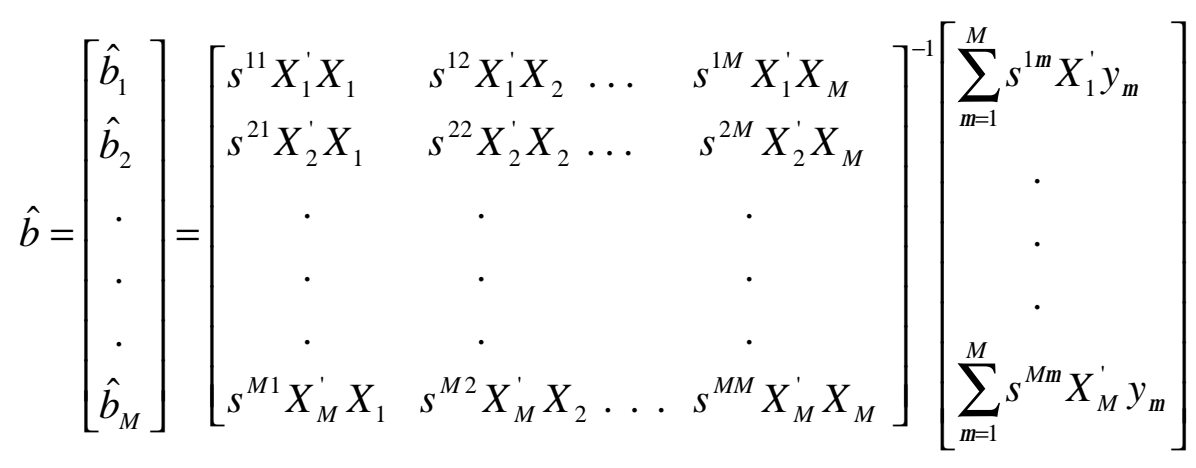

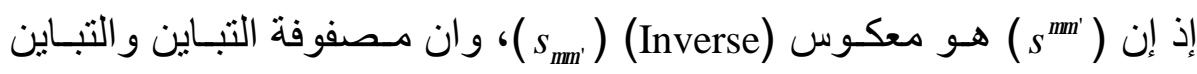

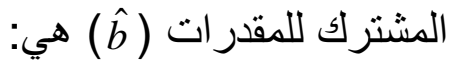

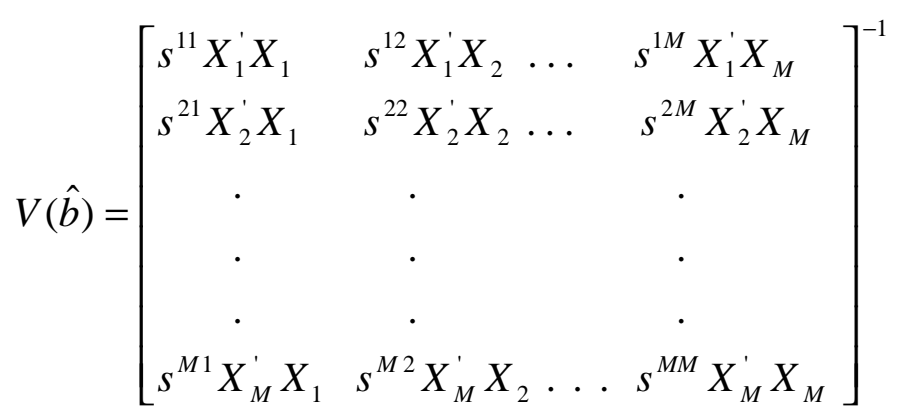

نلاحظظ ممـا تقدم أن الحجـر الأسـاس لطريقـة (SUR) أن هنـاك ارتباطـاً بـين

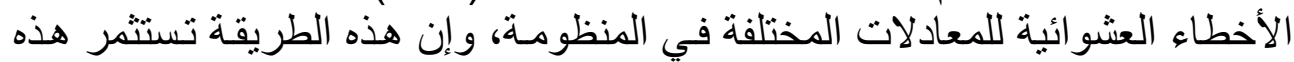

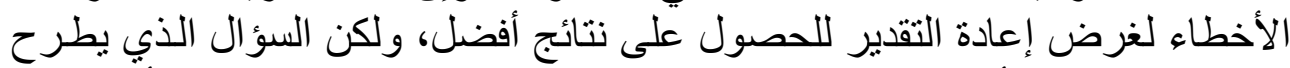

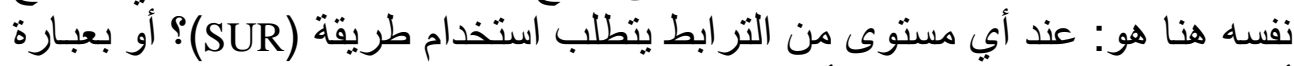

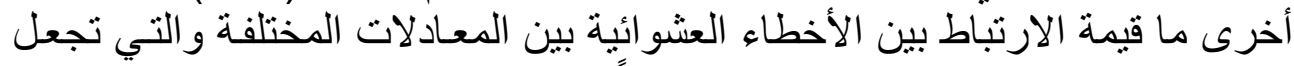

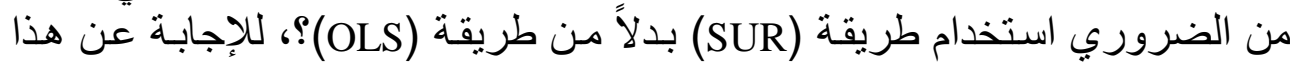

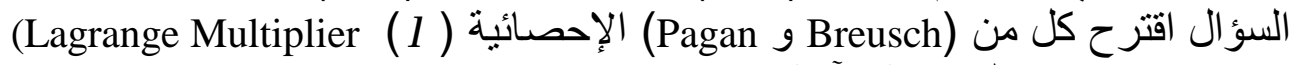

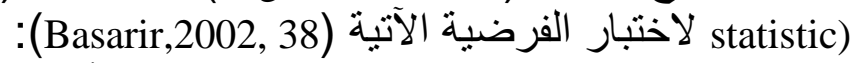
فرضية العدم Ho: إن مصفوفة التغاير لحدود الخطأ العشو ائي لمعادلات المنظومة هي مصفو فة قطرية.

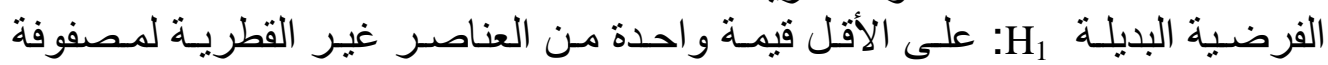

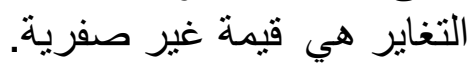

وتأخذ ( ل الصيغة الآتية:

$\lambda=T \sum_{\mu=2}^{M} \sum_{j=1}^{\mu-1} r_{i j}^{2}$ 


\section{يحيى[rrr]}

تمثل (T) عدد المشاهدات، ويمثل ( المختلفة في المنظومة ويأخذ الصيخة الآتية: $r_{\mu j}^{2}=\frac{s_{\mu j}^{2}}{s_{\mu \mu} s_{j j}}$

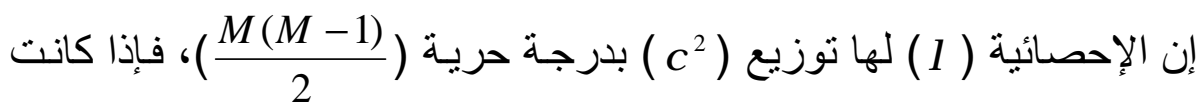

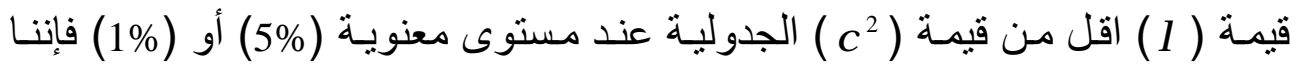

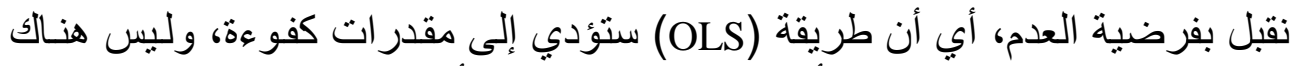
حاجة لتطبيق طريقة (SUR). أما إذا كانت قيمة (

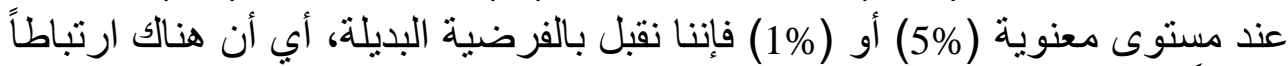

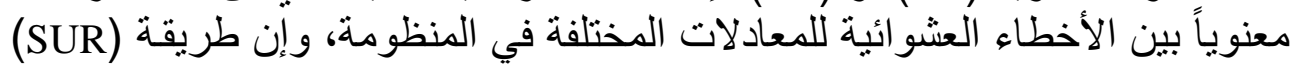
ستؤدي إلى مقدر ات أكثر كفاءة من مقدرات طريقة (OLS).

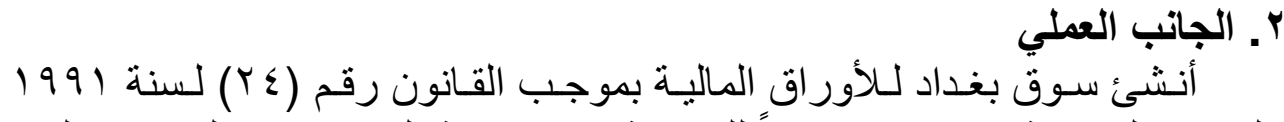

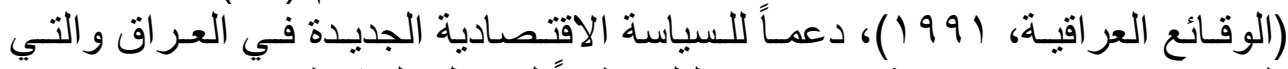

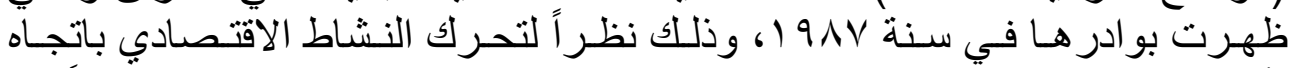

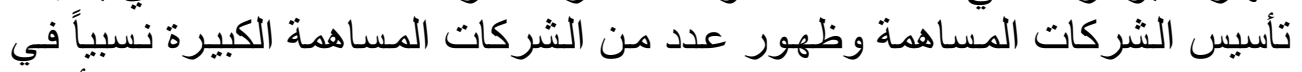

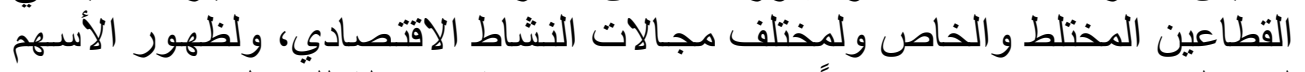

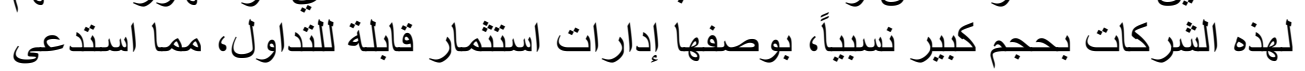

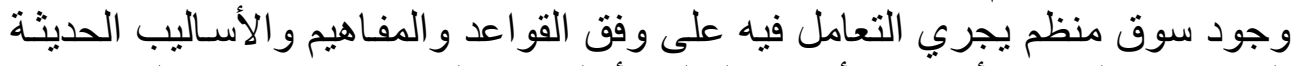

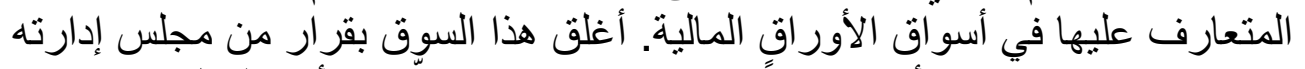

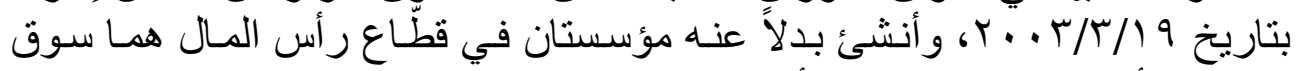

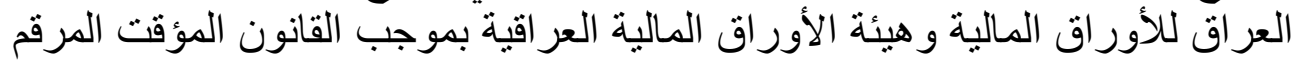
Y...

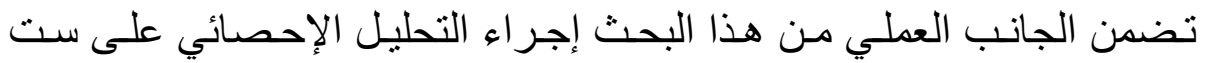

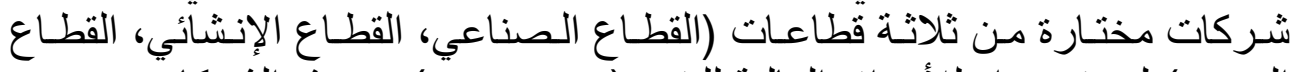

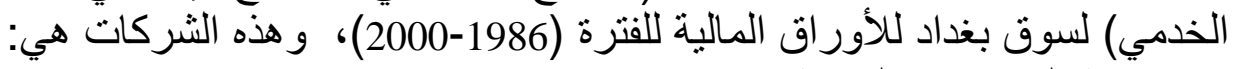
ا ـ ـ شركة الصناعات الخفيفة.

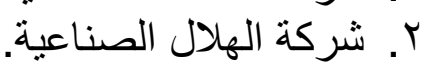

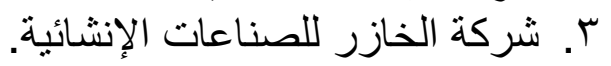
ع. شركة الصناعات الإنشائية الحديثة.

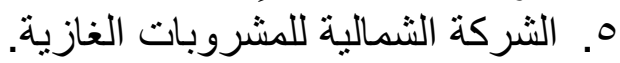
7. شركة الصناعات الإلكترونية.

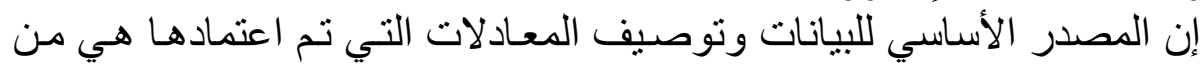

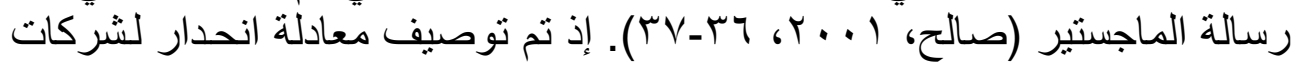


مختـارة مـن شـركات سـوق بغداد للأوراق المالية للفترة مـن (1986-2000) وكمـا

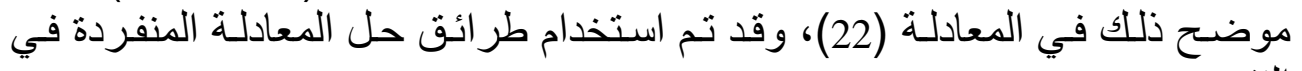
التقدير:

$Y_{i}=b_{0}+b_{1} X_{1 i}+b_{2} X_{2 i}+b_{3} X_{3 i}+b_{4} X_{4 i}+b_{5} X_{5 i} \quad i=1,2,3,4,5,6 \quad \ldots$

i : أسماء الثركات وحسب التسلسل الوارد أعلاه : i 每: هو وثيقة يمثل حقاً عينياً أو نقدياً في رأس مـال الشركة قابل للتداول (للبيع و الثر اء) ويعطي صاحبه حقوقاً خاصة.

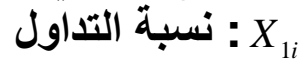

تعد نسبة التداول من أهم الطر ائق المستخدمة في تحديد رأس المـال العامل

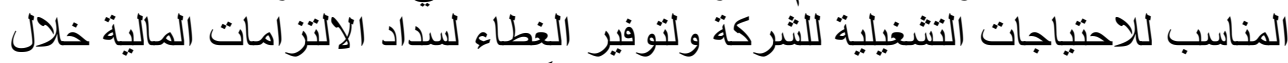

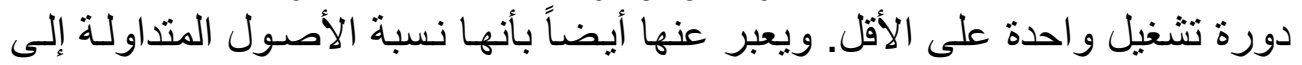

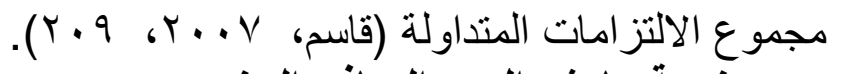
ن : ن $X_{2 i}$

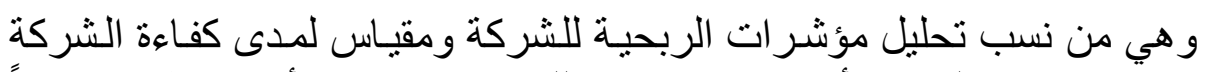

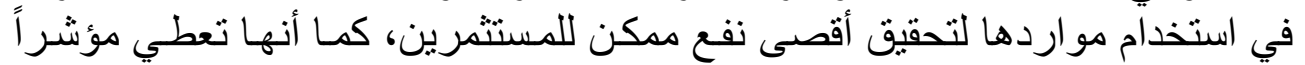

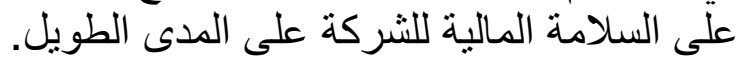
X

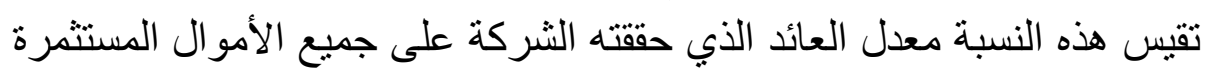

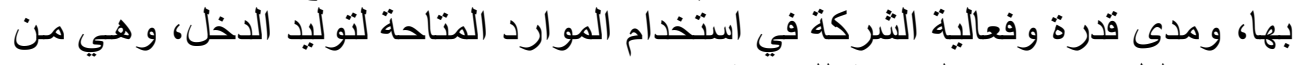
نسب تحليل مؤشر ات الربحية للشركة.

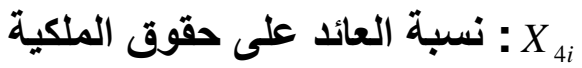

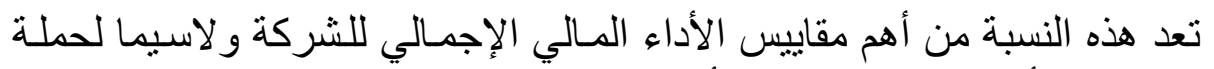

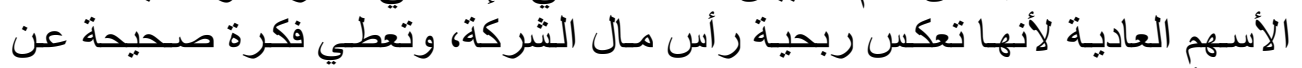

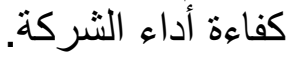

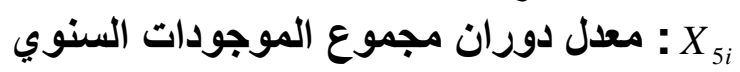

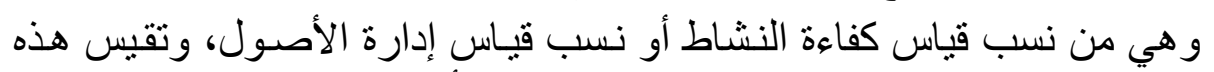

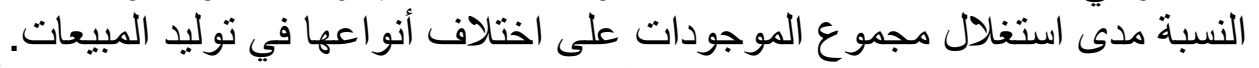

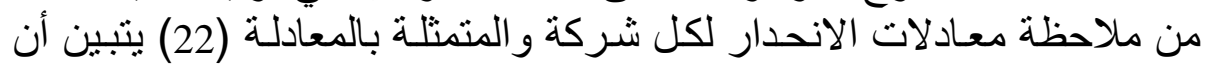

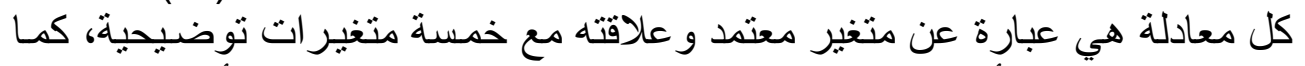

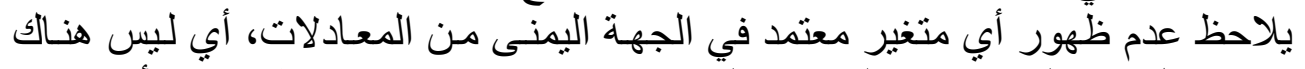

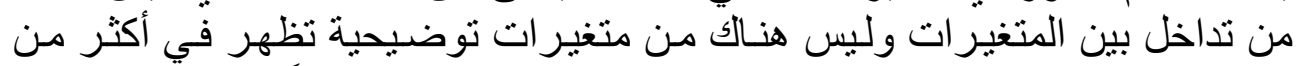

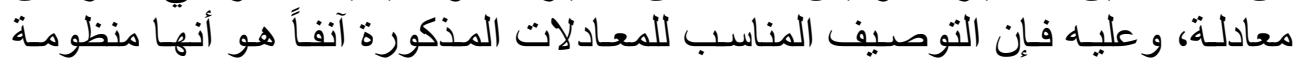




\section{يحيى [rro]}

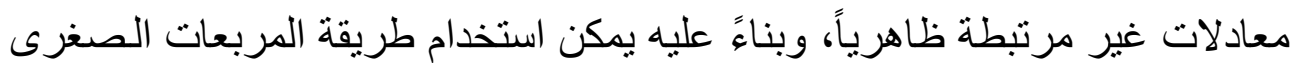

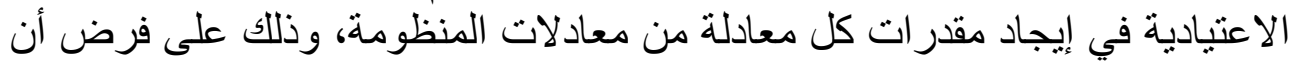

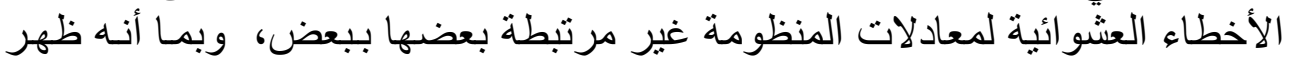

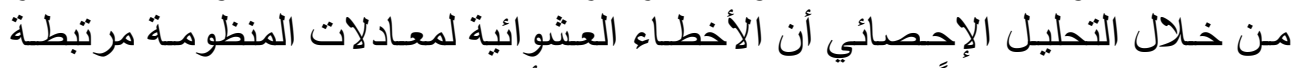

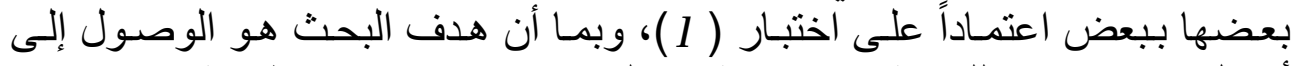

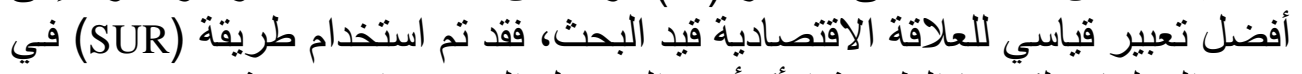
تقدير المعلمات لكونها الطريقة الأكفأ في الوصول فيدة إلى مقدر ات منسقة و غير متحيزة (Consistent \& Unbiased Estimator) استخدام البرنامج الإحصائي الجاهز (SAS.9).

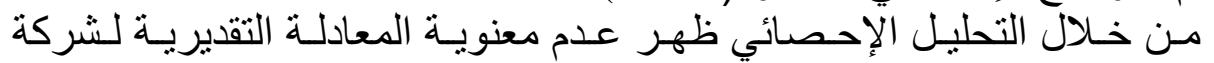

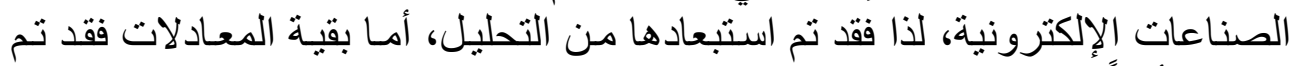

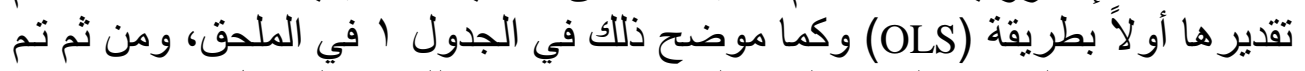

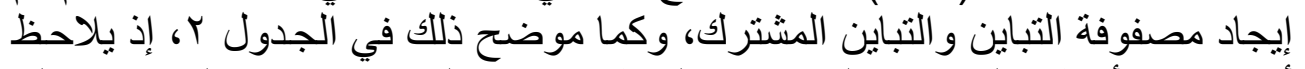

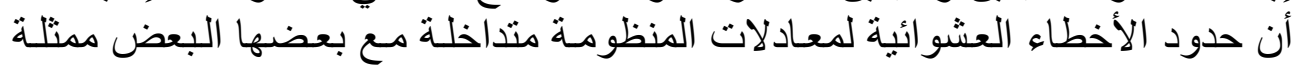

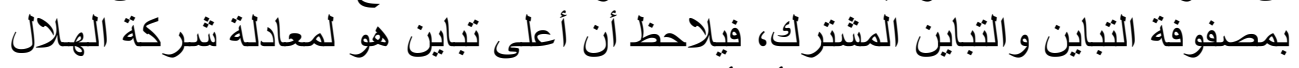

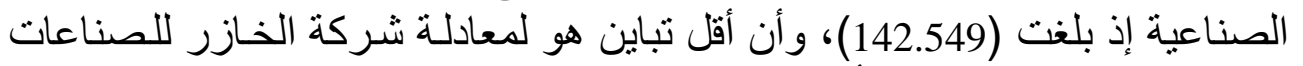

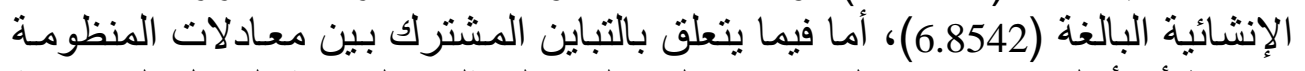

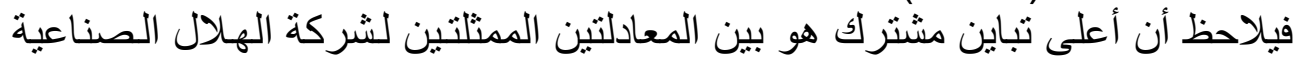

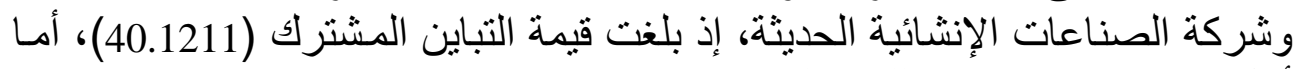

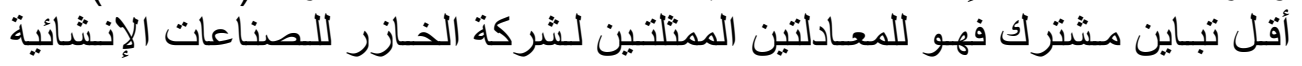

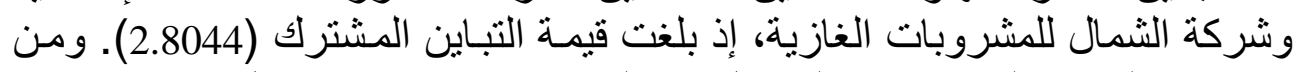

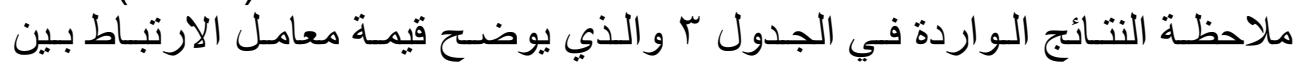

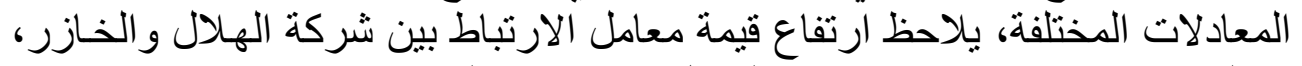

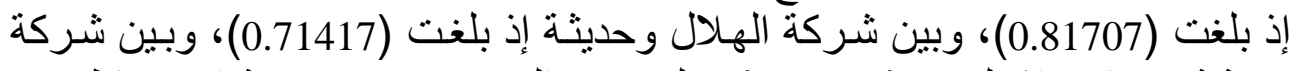

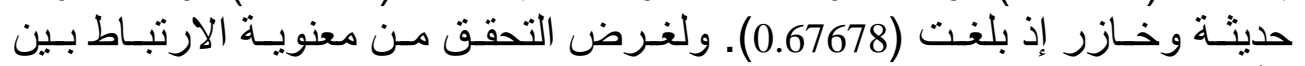

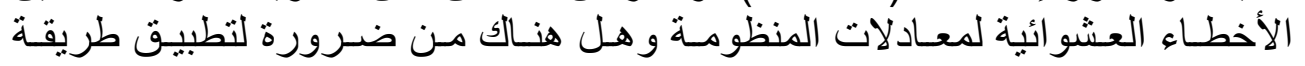
(SUR)

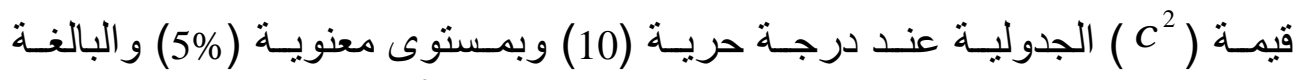

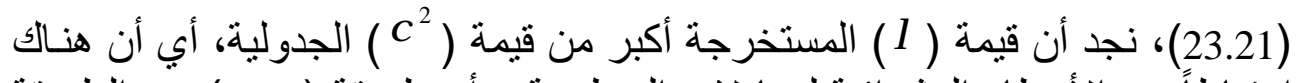

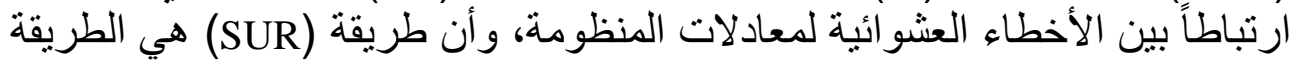
الملائمة للتقدير.

من ملاحظة نتائج انحدار المعادلات الواردة في الجدول ا و المقدرة بطريقة ومقارنتها مع النتائج الواردة في الجدول ه و والمقدرة بطريقة (OLS)

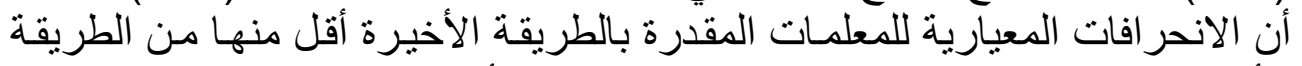

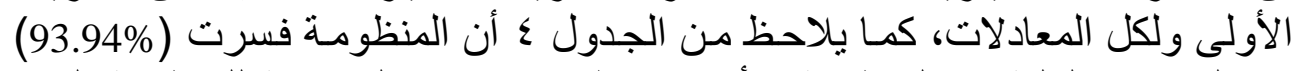

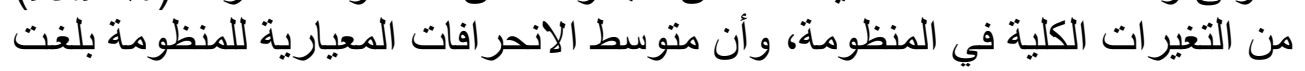




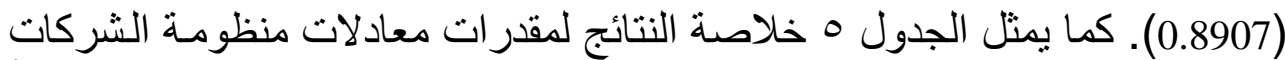

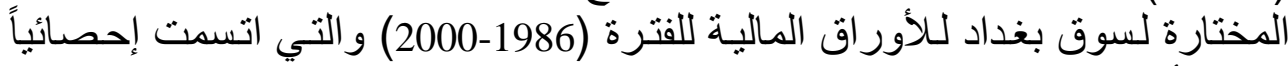

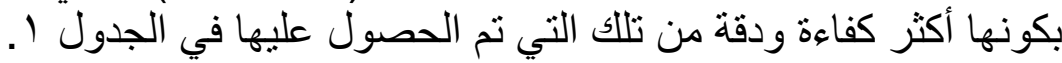

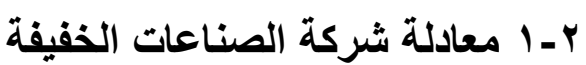

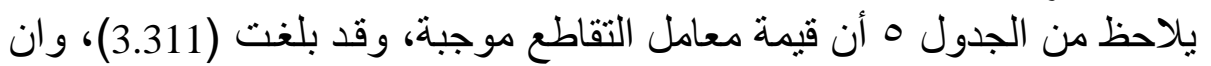

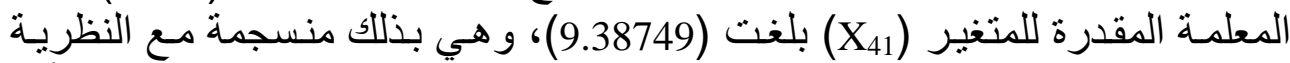

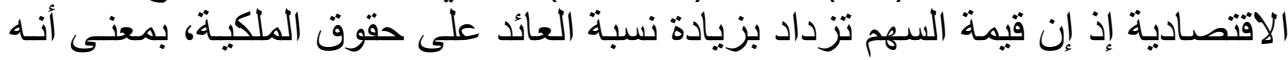

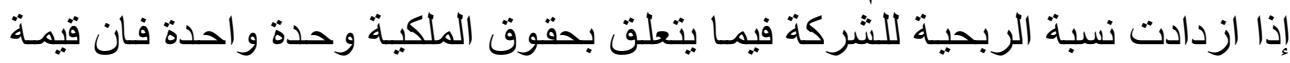

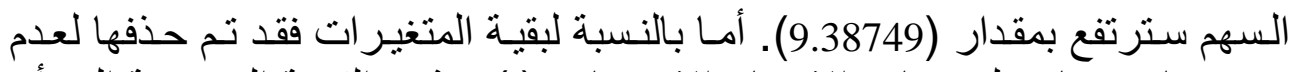

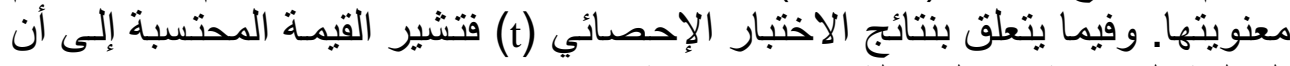
المعلمة التقديرية في المعادلة تتمتع بمعنوية عند مستوى (1) (0.05).

r r-r بعادلة شركة الهلال الصناعية

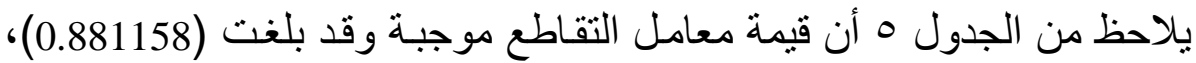

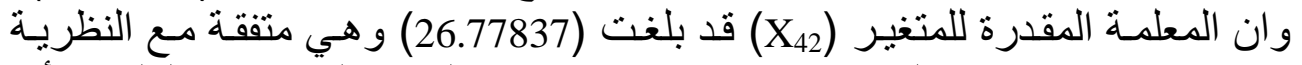

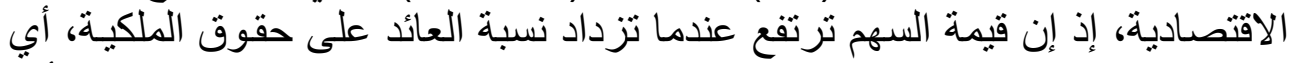

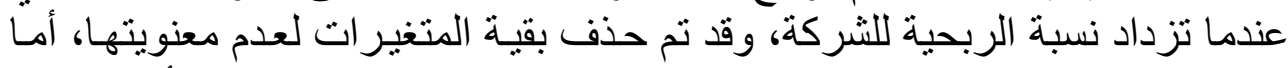

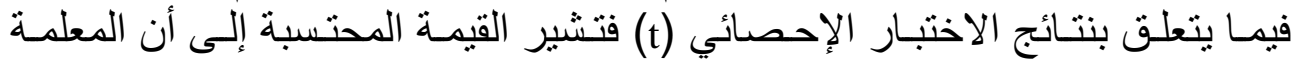
التقديرية في المعادلة تتمتع بمعنوية عند مستوى (t) (0.01).

r r r بعادلة شركة الخازر للصناعات الإنشائية

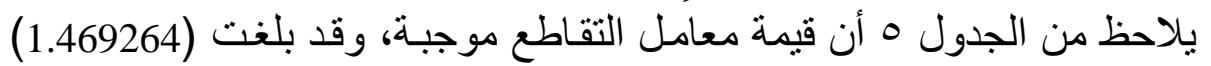

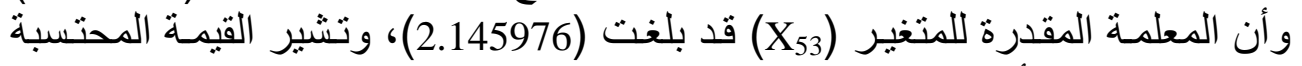

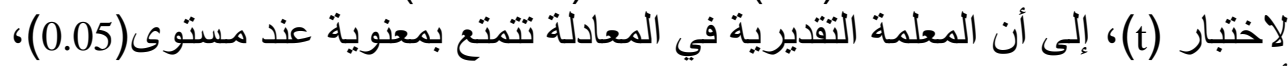

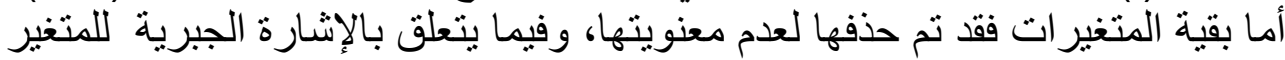

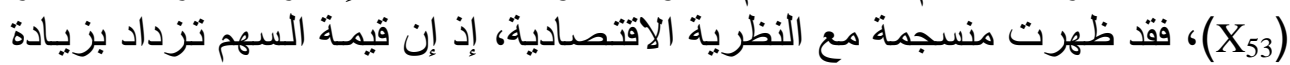

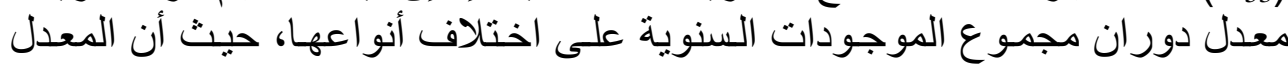
المرتفع يدل على حسن استغلال موجودات الثرات الثركة في زيادة المبيعات.

r ب- بـ معادلة الثركة الإنثائية الحديثة

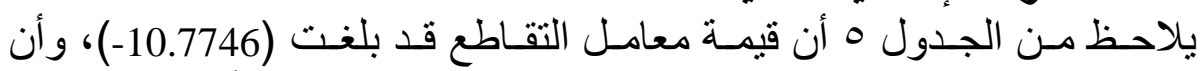

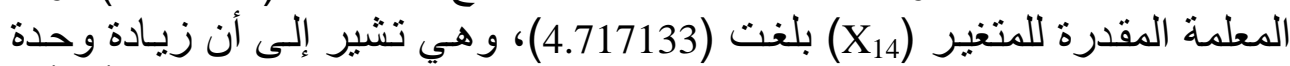

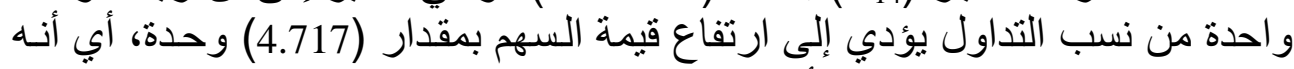

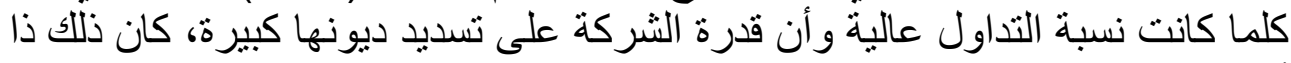

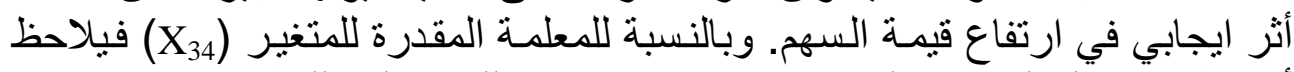

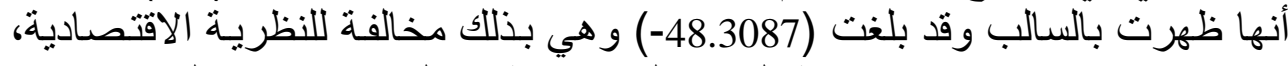
إذ إن زيادة وحدة واحدة في نسبة العائد على الاستثمار السنوي يؤدي إلى الخفاض 


\section{يحيى [rrV}

في قيمة السهم بمقدار (48.3087-)، وربمـا يعود السبب في ذلك إلى مـا تمثلـه نسبة

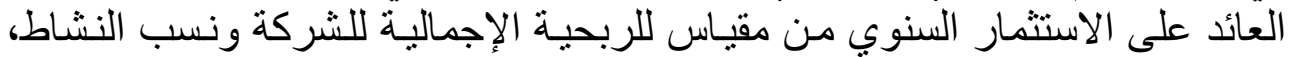

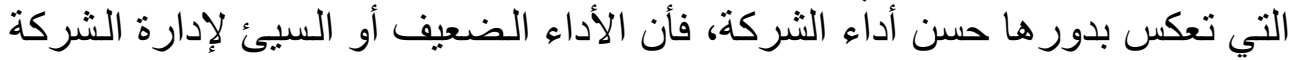

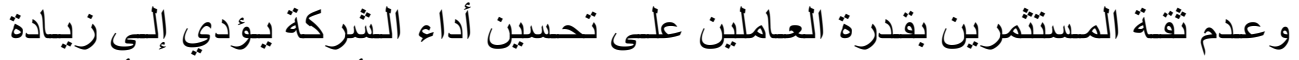

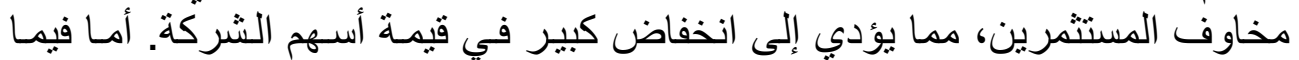

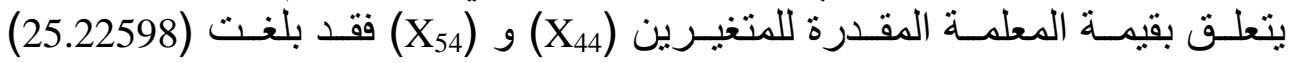

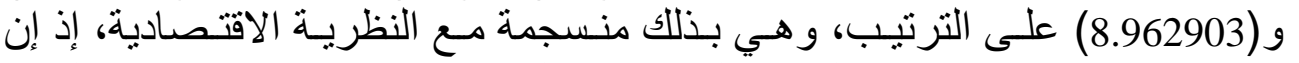

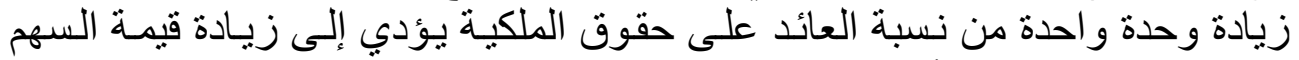

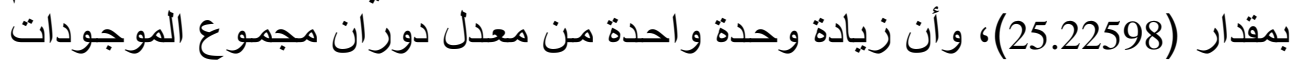
السنوي يؤدي إلى زيادة في قيمة السهم بمقدار (2258) (8.962903).

ץ ـ- معادلة الشركة الشمالية للمشروبات الغازية

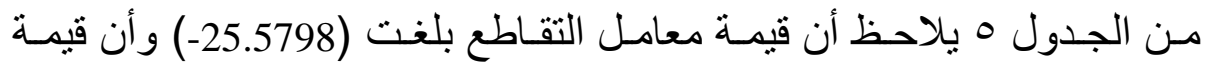

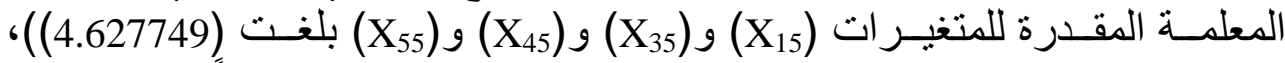

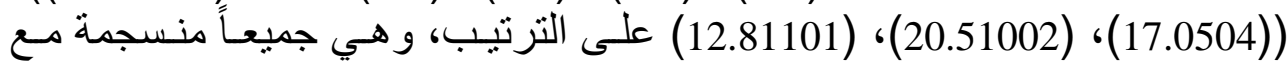

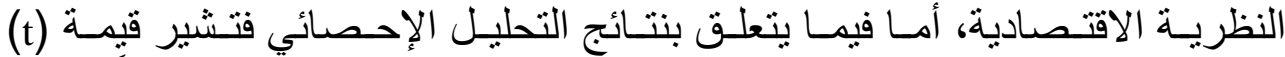

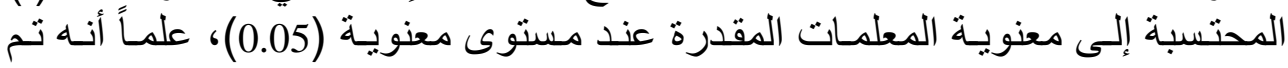
حذف المتغير (X25) لعدم معنويته.

الاستنتاجات

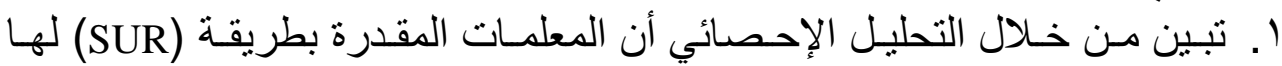

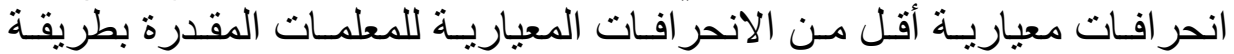

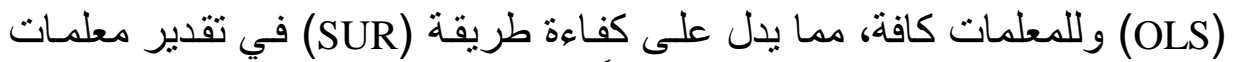

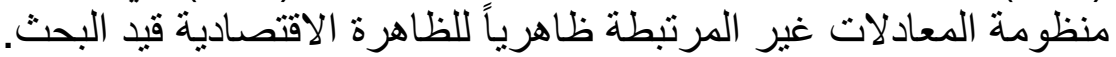

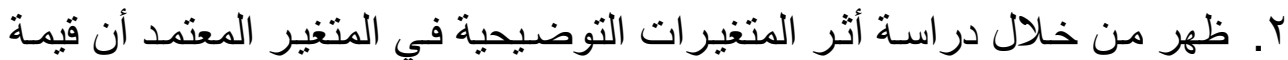

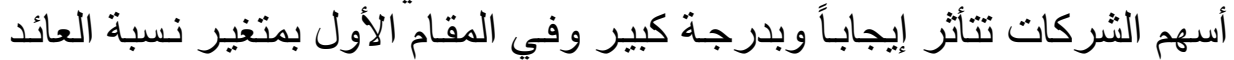

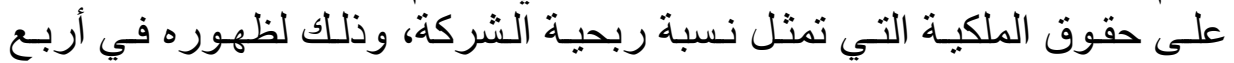

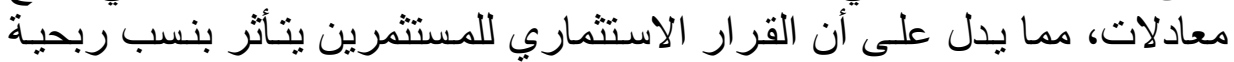

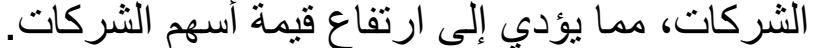

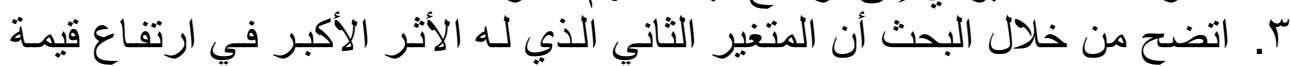

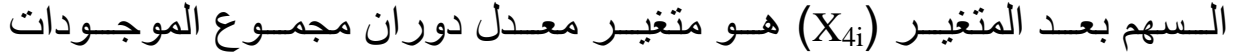
السنوي(X).

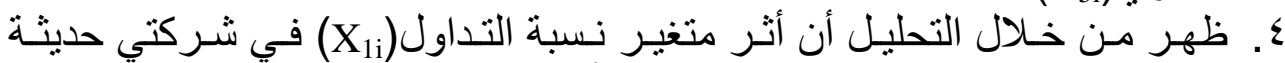
و المشروبات الغازية له القيمة نفسها تقريباً.

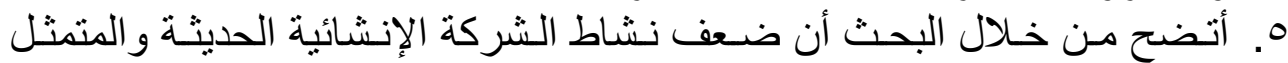

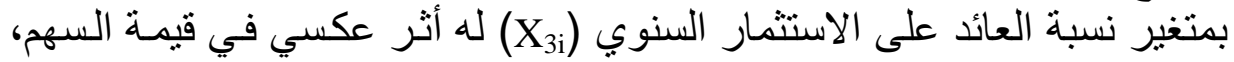

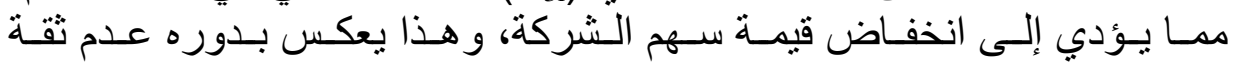




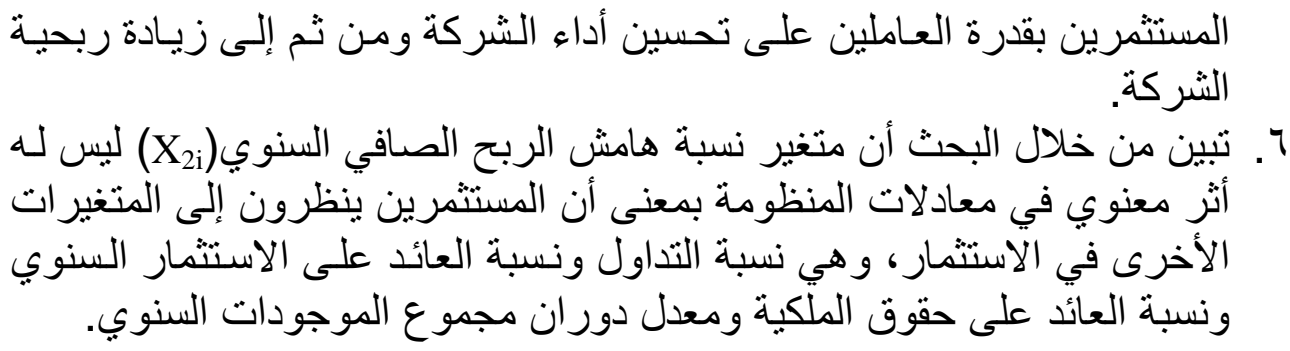

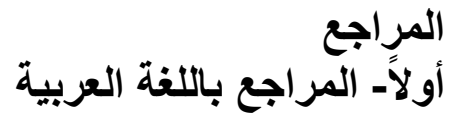

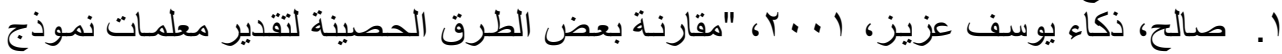

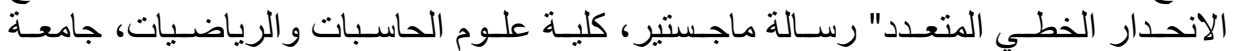

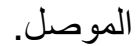

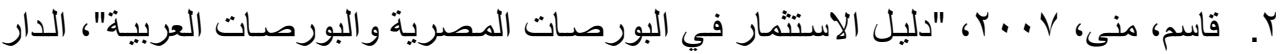

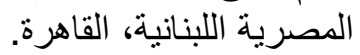

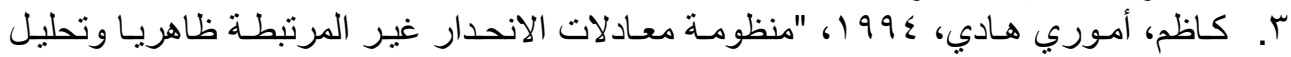

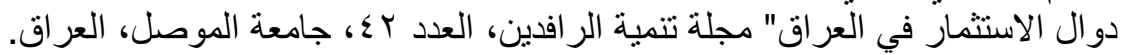

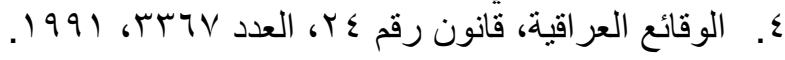

ثانياً- المراجع باللغة الاجنبية

1. Basarir,A.,2002" Multidimensional Goals of Farmers in the Beef Cattle and Dairy" In partial fulfillment of the requirements for the degree of Doctor of Philosophy, Agricultural and Mechanical College, Louisiana State University.

2. Intrilligator, Michael D.1996." Econometrics Models, Techniques and Applications", Prentice Hall.

3. Mardia K.V. \& Kent J.T. \& Bibby J.M. 1980 "Multivariate Analysis" Academic Press Inc. London.

4. Pindyck R.S. \& Rubinfeld 1981, "Econometric Models and Economic Forecasts", McGraw-Hill International Book Co. New York.

5. SAS/ETS User's Guide 1999,Version (9) ,SAS Institute Inc., Cary, North Carolina, USA.

6. Zellner,A., 1962"An Efficient Method of Estimating Seemingly Unrelated Regressions and Test for aggregation bias", JASA, Vol.57. 